\title{
Emission and Absorption in the M87 LINER ${ }^{1}$
}

\author{
Bassem M. Sabra, ${ }^{2,3}$ Joseph C. Shields, ${ }^{2}$ Luis C. Ho, ${ }^{4}$ Aaron J. Barth, ${ }^{5,6}$ and Alexei V. \\ Filippenko ${ }^{7}$
}

\begin{abstract}
The nucleus of M87 displays a LINER spectrum at optical wavelengths, with a nuclear disk of nebulosity that is resolved by the Hubble Space Telescope. We present new results from optical and ultraviolet spectra of the central $\sim 40 \mathrm{pc}$ as measured by HST. In contrast with previous results for the extended disk, the emission-line spectrum of the central region is best reproduced by a multi-component photoionization scenario, rather than shock heating. The nebular properties as well as energetic considerations suggest a transition on scales of several tens of parsecs, from predominantly photoionization by a central accretion source, to shock ionization within the disk. If this source is at all representative, it suggests that many LINERs may be composite in terms of the energetic processes that give rise to the emission spectrum. We also report measurements of resonance-line absorption for the nucleus. The absorption spectrum, like the emission lines, is characterized by low ionization. The absorption line measurements coupled with independent constraints suggest a total hydrogen column density of $10^{19}-10^{20} \mathrm{~cm}^{-2}$, outflowing from the galaxy center with a velocity of $\sim 126 \mathrm{~km} \mathrm{~s}^{-1}$. The kinematic signature of an outflow, along with evidence that the absorber covers the power-law continuum source but not the emission-line clouds, suggests that the absorbing matter is related to accretion phenomena in the nucleus. The presence of such an outflow resembles similar behavior in luminous AGNs, although the low ionization that characterizes LINERs is probably indicative of a different mode of accretion in these sources.
\end{abstract}

Subject headings: galaxies: individual (M87) — galaxies: nuclei — galaxies: active

\footnotetext{
${ }^{1}$ Based on observations with the NASA/ESA Hubble Space Telescope obtained at the Space Telescope Science Institute, which is operated by the Association of Universities for Research in Astronomy, Inc., under NASA contract NAS5-26555.

${ }^{2}$ Department of Physics \& Astronomy, Ohio University, Athens, OH 45701.

${ }^{3}$ Department of Astronomy, University of Florida, Gainesville, FL 32611.

${ }^{4}$ The Observatories of the Carnegie Institution of Washington, 813 Santa Barbara St, Pasadena, CA 91101.

${ }^{5}$ Hubble Fellow.

${ }^{6}$ Department of Astronomy, California Institute of Technology, 105-24, Pasadena, CA 91125.

${ }^{7}$ Department of Astronomy, University of California, Berkeley, CA 94720-3411.
} 


\section{INTRODUCTION}

A large fraction of nearby galaxies harbor low-ionization nuclear emission-line regions (LINERs; e.g., Heckman 1980; Ho, Filippenko, \& Sargent 1997). A variety of mechanisms have been suggested for the underlying power source in these objects, including shocks, black hole accretion, and hot stars. LINER case studies have revealed candidate prototypes for each of these phenomena, strengthening speculation that LINERs are a heterogeneous class (e.g., Filippenko 1996).

The LINER galaxy M87 is a particularly interesting example. Ground-based optical spectra of its nucleus show narrow-line emission with a complex velocity profile, but no clear signature of the broad permitted lines that are the hallmark of quasar activity. Observations with the Hubble Space Telescope (HST) reveal a spatially resolved disk of emission at the nucleus, and analysis of emission from the disk provides strong evidence of shock excitation (Dopita et al. 1997, hereafter D97). Considered alone, these properties give little indication that the system is related to luminous AGNs; yet this object provides one of the best cases for accretion-powered activity in the nucleus of a galaxy. The evidence takes the form of its famous synchrotron jet (e.g., Boksenberg et al. 1992; Bicknell \& Begelman 1996) and a velocity field in the nuclear gas disk requiring the presence of a central dark object with a mass of $3.2 \times 10^{9} \mathrm{M}_{\odot}$ (Ford et al. 1994; Harms et al. 1994; Macchetto et al. 1997).

One possible interpretation of the existing results for M87 is that LINER emission is caused primarily by shocks, even when coexistent with an accretion power source that may generate substantial ionizing radiation. This scenario contrasts markedly with the behavior of Seyfert galaxies, which have narrow-line emission powered primarily by photoionization (e.g., Laor 1998). Moreover, the evidence for shocks in the M87 disk comes from ultraviolet emission-line ratios, but the same diagnostics observed in a handful of other LINERs do not provide similar indications of mechanical heating (e.g., Barth et al. 1996, 1997; Maoz et al. 1998). The latter finding may imply that the LINER in M87 is an anomaly; however, another possibility is that the uncharacteristic result for this source reflects the unusual circumstance that its central nebulosity is resolved, and the previous HST study considered emission from only a part of the disk that did not include the nucleus per se. In order to understand fully the nature of the nebulosity at the center of M87, and to assess the larger implications for LINERs, measurement of the UV/optical spectrum of the nucleus is clearly

desirable. In this paper we present the results of spectroscopic observations carried out with HST for this purpose.

\section{Observation And Data Analysis}

Long-slit spectra of the nucleus of M87 were obtained with the Space Telescope Imaging Spectrograph (STIS) on 1999 February 1 UT, using the $52^{\prime \prime} \times 0^{\prime \prime} 5$ aperture and the gratings listed

in Table 1. The slit was oriented at position angles $216^{\circ}$ and $240^{\circ}$ for the optical (CCD) and UV (MAMA) data, respectively. (The difference in position angle resulted unintentionally from a 
roll-angle adjustment during the observation sequence.) The resulting spectra provide continuous wavelength coverage of the nucleus in the range $\sim 1150-10270 \AA$. The relatively large slit width leads to rather low spectral resolution (see Table 1) but was selected in order to maximize the measured signal in the UV bandpass, while maintaining a uniform aperture dimension across the entire spectrum. The full-width at half maximum (FWHM) spatial resolution is $\sim 0$ '.05 for the MAMAs, and $\sim 0$ "' 12 for the CCDs. The data were calibrated "On-The-Fly" upon retrieval from the data archive at the Space Telescope Science Institute. All data reduction and analysis was performed with $\mathrm{IRAF}^{8}$. With this slit width, geocoronal Ly $\alpha$ is blended with the redshifted Ly $\alpha$ from M87. We measured the background geocoronal emission in the G140L spectrum near the ends of the slit, and subtracted the result from each row of the two-dimensional spectrum, making sure that we also propagate errors in the correct way.

Spectra of the nucleus were extracted for an aperture size of $\sim 00^{\prime} 25$ along the slit (i.e., 10 MAMA pixels and $5 \mathrm{CCD}$ pixels, corresponding to approximately twice the spatial FWHM for the CCD). The result is shown in Figure 1. In order to aid in removing any underlying galaxy starlight, we generated a stellar continuum template by averaging the spectra of off-nucleus regions that were emission-free. The resulting aperture was $10^{\prime \prime}$ ( $5^{\prime \prime}$ for UV spectra) wide and centered at $7{ }^{\prime \prime} .5$ (3.'75 for UV spectra) southwest of the nucleus. Emission from the nebular disk at these scales is negligible within our aperture, in comparison with that measured from the nucleus. HST images show a rapid fall-off of nebular surface brightness with radius (e.g., D97). In contrast with the FOS study reported by D97, which measured the spectrum 0.6 from the nucleus with a $1{ }^{\prime \prime} 0$ aperture, the data reported here employ a narrower slit, and consequently do not exhibit a signal-to-noise ratio sufficient to measure nebular features outside the central aperture defined above.

We measured the fluxes of the emission features while simultaneously fitting the continuum, via $\chi^{2}$ minimization, using SPECFIT as implemented in IRAF (Kriss 1994). We fitted Gaussians to the emission lines, and represented the continuum as the sum of a featureless power law and the galaxy template. The results of the measurements are given in Table 2 . In all cases, the fit results were consistent with the continuum being dominated by the power-law component. Errors were evaluated by SPECFIT from the accompanying error extensions to the spectra. The FWHM velocity for $\mathrm{H} \beta$ is $\sim 2100 \mathrm{~km} \mathrm{~s}^{-1}$, after correcting for the instrumental profile for a point source, and $\sim 900 \mathrm{~km} \mathrm{~s}^{-1}$ if the emission is extended uniformly across the slit; the latter value can thus be taken as a lower limit. Due to the significant intrinsic velocity width and the low spectral resolution, $\mathrm{H} \alpha$ was heavily blended with [N II] $\lambda \lambda 6548,6583$, and the [S II] $\lambda \lambda 6717,6731$ lines were also heavily blended with each other; no attempt was made at deblending these composite features, which we instead fit by single Gaussians. The width and redshift of the [O I] $\lambda 6364$ template were linked to those of [O I] $\lambda 6300$ and, as dictated by atomic physics (Osterbrock 1989), [O I] $\lambda \lambda 6300 / 6364=3.0$. The same procedure was followed for [O III] $\lambda \lambda 4959,5007$.

\footnotetext{
${ }^{8}$ IRAF is distributed by the National Optical Astronomy Observatories, which are operated by the Association of Universities for Research in Astronomy, Inc., under cooperative agreement with the National Science Foundation.
} 
A joint analysis of the optical and UV spectra is potentially sensitive to reddening. The fact that $\mathrm{H} \alpha$ was heavily blended with the [N II] lines makes it impossible to get a direct measure of reddening using the $\mathrm{H} \alpha / \mathrm{H} \beta$ ratio. Moreover, $\mathrm{H} \delta$ is blended with [S II] $\lambda \lambda 4069$, 4076, and $\mathrm{H} \gamma$ leads to unphysical extinction values when combined with $\mathrm{H} \beta$; this may be due to an overestimate of the $\mathrm{H} \gamma$ flux caused by blended emission in [O III] $\lambda 4363$. Our spectral range includes the recombination lines He II $\lambda 4686$ and He II $\lambda 1640$. For Case B recombination, the ratio He II $\lambda \lambda 1640 / 4686 \approx 7$ (Hummer \& Storey 1987). Our spectra set a $3 \sigma$ upper limit on He II $\lambda 4686$ of $20 \%$ of $\mathrm{H} \beta$. This limit is calculated according to the formula $3 \sqrt{2 \pi} \sigma_{c}(14 \AA)$, where $\sigma_{c}$ is the root-mean-square uncertainty per Angstrom in the local continuum, and $14 \AA$ is the measured Gaussian dispersion of the $\mathrm{H} \beta$ profile. (This prescription corresponds to a Gaussian profile with amplitude $3 \sigma_{c}$.) The result is (He II $\lambda \lambda 1640 / 4686)_{\text {observed }}>2.0$. Employing the extinction curve of Cardelli, Clayton, \& Mathis (1989), we find that $A_{V}<2.4 \mathrm{mag}$. The Galactic foreground extinction toward M87 is expected to be much less than this value, and independent estimates of the total absorption (§3) suggest that the Galactic component dominates the total extinction, with $A_{V} \approx 0.15$ mag.

Additional spectra of the nucleus of M87 spanning 1140 - $6820 \AA$ were obtained on 1996 July 2 UT with the FOS, using the $0^{\prime} \cdot 21$ aperture (Table 1). The calibrated data were obtained from the public domain archive. We measured the emission lines as for the STIS spectra, assuming a power-law form for the continuum. Uncertainties in the line fluxes are generally larger for the FOS observations, reflecting a somewhat lower signal-to-noise ratio. Measurements from the two spectra are in general agreement although significant differences are present in Ly $\alpha$, which may be affected by uncertain geocoronal subtraction for the FOS spectrum, and [Ne III] $\lambda 3869$.

In addition to emission lines, there is a wealth of UV absorption features seen in the spectra. The velocity shifts of some lines are consistent with absorption within the Galaxy, while others appear associated with M87. We used spectra with the highest spectral resolution for our absorption-line measurements. Table 1 shows that all the FOS gratings have higher spectral resolution than those employed with STIS, except for FOS G160L, which has a lower resolution than STIS G140L. The absorption lines in the FOS spectra segregate into two systems distinguished by their line widths; those with FWHM $\approx 200 \mathrm{~km} \mathrm{~s}^{-1}$ are of Galactic origin (and are unresolved), while those with FWHM $\approx 400 \mathrm{~km} \mathrm{~s}^{-1}$ are intrinsic to M87, where the FWHM values quoted here have not been corrected for the instrument profile of $\mathrm{FWHM}=230 \mathrm{~km} \mathrm{~s}^{-1}$. The absorption lines in the STIS G140L have FWHM $\approx 400 \mathrm{~km} \mathrm{~s}^{-1}$, uncorrected for the line-spread function of $\mathrm{FWHM}=190 \mathrm{~km} \mathrm{~s}^{-1}$ for a point source; the velocity widths of many features in this spectrum are potentially affected by blends. Identification of features was based on wavelength correspondence with line lists from Verner, Barthel, \& Tytler (1994), and consistency tests with the measured line strengths or upper limits for resonance features from the same ions.

The STIS and FOS observations are separated by $\sim 2.6$ years. The equivalent widths of the absorption lines agree to within the measurement errors. The continuum level in the FOS spectrum is higher by $20 \%$, with greater contrast at shorter wavelength. Tsvetanov et al. (1998) discussed variability in the optical continuum during 1994 and 1995, and found differences of a factor of $\sim 2$ 
on a timescale of 2.5 months, so variability is clearly a concern when comparing measurements from different epochs.

\section{Results AND Interpretation}

The physical interpretation of nebular emission in LINERs is complicated by the fact that several different energy sources may give rise to such emission. Theoretical predictions of optical lines are degenerate in the sense that the same line ratios can be explained equally well by more than one physical scenario, including in particular shocks and photoionization (e.g., D97; Allen, Dopita, \& Tsvetanov 1998). Allen et al. (1998) have suggested that the inclusion of ultraviolet emission diagnostics provides a basis for distinguishing between shocks and photoionization, and UV emission lines figure prominently in the analysis by D97 implicating shocks in the extended nuclear disk in M87. In the present study we extend this analysis to the interpretation of the emission of nuclear emission as measured in the central $\sim 00^{\prime \prime} 5$, corresponding to $\sim 39 \mathrm{pc}$ (for an assumed distance of $16.1 \mathrm{Mpc}$; Tonry et al. 2001).

\subsection{Line Ratio Diagrams and the Excitation Mechanism}

Comparisons of our measurements with theoretical predictions are presented in two-dimensional line ratio diagrams in Figure 2, which employs optical and UV lines, and Figure 3, which uses exclusively UV lines. These diagrams are selected from those shown by D97 and Allen et al. (1998) to hold promise for distinguishing ionization processes. In addition to our STIS results, line ratios from the FOS spectra of the nucleus (our measurements) and of the ionized disk (D97) are also shown. The diagrams involve intensity ratios of lines close in wavelength, so that they are relatively insensitive to reddening; the plotted data points have consequently not been corrected for reddening.

Predictions for several excitation mechanisms are overplotted in Figures 2 and 3. Predicted ratios for shock excitation are taken from Dopita \& Sutherland (1995); for their models, the parameters that produce the range in line ratios are the shock velocity and the magnetic parameter $B / n^{1 / 2}$, where $B$ is the magnetic field strength and $n$ is the hydrogen number density. Predictions for photoionized plasma assume power-law continua with a spectral index $\alpha=(-1.0,-1.4)$ (assuming $\left.f_{\nu} \propto \nu^{\alpha}\right)$, density $n_{H}=(100,1000) \mathrm{cm}^{-3}$, and ionization parameter $U=10^{-4}-10^{-1}$, where $U$ is the ratio of ionizing photon and hydrogen densities at the face of the irradiated cloud (Binette, Wilson, \& Storchi-Bergmann 1996).

A possible limitation of these photoionization models is their assumption of a single population of ionization-bounded clouds. Binette et al. (1996) have argued that the spectra of AGN narrowline regions can be better reproduced by photoionization scenarios with multi-component cloud systems; in particular, they emphasize the possible importance of a mix of ionization- and matter- 
bounded clouds. We have consequently also shown for comparison in Figures 2 and 3 the predictions of a two-zone photoionization model, in which we have reproduced the Binette et al. results using the photoionization code CLOUDY, version 90.05 (Ferland et al. 1998). In this scenario, matterbounded (MB) clouds filter the ionizing continuum from the AGN before it irradiates the ionization bounded (IB) clouds. The MB clouds are irradiated by a power law with $\alpha=-1.3$, and are characterized by $U_{M B}=0.04$ and $n_{M B}=50 \mathrm{~cm}^{-3}$, while the IB clouds have $U=5.2 \times 10^{-4}$ and $n_{I B}=2300 \mathrm{~cm}^{-3}$. The relative contribution of emission from the two cloud populations is parametrized by $A_{M / I}$, which is the ratio of solid angles subtended by MB and IB clouds. Formally, $A_{M / I}$ is always larger than one, but in calculating the resulting spectrum, it can be effectively less than one if the MB clouds are hidden along our line of sight but seen by the IB gas.

The measured ratios reported by D97 at an off-nuclear position, and for the nucleus as given here, are different, and Figures 2 and 3 suggest different interpretations for the two locations. A similar inference was noted by Sankrit, Sembach, \& Canizares (1999) based on FOS measurements of the C IV/Ly $\alpha$ ratio alone. As discussed by D97 and illustrated also here, the line ratios for the off-nucleus measurement of M87 are consistent in all of these diagrams with a shock interpetation; the photoionization predictions, particularly the single cloud models, do not agree with the data. The line ratios measured for the M87 nucleus are, however, less clear-cut in their interpretation. In Figure 2 (optical and UV lines), the nucleus ratios generally fall within the shock model loci and are inconsistent with both single-cloud and $A_{M / I}$ photoionization predictions. However, in Figure 3 (UV lines) the nucleus measurements fall outside the predictions for shocks. Single-cloud photoionization models are likewise inconsistent with these data; but the $A_{M / I}$ sequence intersects the measured ratios in all of the plots in Figure 3, and in most cases the data are in fact consistent with predictions for a single value of $A_{M / I} \approx 0.03$. This result provides a strong suggestion that photoionization is, in fact, important in the nucleus. In contrast, comparison of the observed ratios with the detailed shock models of Dopita \& Sutherland (1995) shows that the optical line ratios in Figure 2 are not reproduced by a single combination of shock parameters. The $A_{M / I}$ model remains problematic, however, for explaining the optical line ratios in Figure 2 as well as generally underpredicting the strengths of the UV lines relative to $\mathrm{H} \beta$ for the nucleus.

Is it possible to reconcile a single excitation scenario with the nucleus line ratios plotted in both Figures 2 and 3? It is interesting to note that the division between optical and UV lines separates strong transitions not only by excitation energy, but also by the critical densities for the collisionally excited lines: lower $n_{\text {crit }}$ for the optical lines, which are forbidden transitions, and higher $n_{c r i t}$ for the UV lines, which are intercombination or resonance features. As a result, increasing the plasma density will affect the predicted line strengths in different ways; the forbidden lines will become suppressed, while the lines with high $n_{\text {crit }}$ will strengthen as they take over a larger share of the nebular cooling. For the M87 nucleus, increasing the nebular density might thus be expected to improve the success of photoionization models by decreasing the $[\mathrm{O} \mathrm{III}] / \mathrm{H} \beta$ ratio and boosting the strengths of the UV lines relative to $\mathrm{H} \beta$. Nebular components spanning a wide range of density are known to exist within AGNs, as revealed by line width- $n_{\text {crit }}$ correlations (e.g., Pelat, Alloin, 
\& Fosbury 1981; Filippenko \& Halpern 1984; Filippenko 1985), and dense components on small spatial scales are explicitly revealed in nuclei through HST studies (e.g., Barth et al. 2001).

To investigate these effects, we experimented with $A_{M / I}$ sequences with increased densities and found that many characteristics of the nuclear spectrum are reproduced if $n_{M B}=10^{6} \mathrm{~cm}^{-3}$ and $n_{I B}=10^{6.3} \mathrm{~cm}^{-3}$. The calculation results are again shown for comparison in Figures 2 and 3. The observed line ratios fall consistently near the predictions for the high- $n A_{M / I} \approx 0.002-0.004$, except in Figure 2a. In the latter case, the predicted [O II] $\lambda 3727$ strength is very weak, due to the low critical density of this line. A likely remedy to this problem is the inclusion of an additional lowdensity IB (low- $n$ IB) component that emits efficiently in this transition while minimally perturbing the other line ratios.

We modeled this third component as the low-density IB of Binette et al. (1996), except that it was irradiated by an ionizing continuum, the same power-law continuum discussed above, which has not been filtered through the MB clouds. To achieve the final combination of high- $n A_{M / I}+$ low- $n$ IB, we assumed that our observation aperture is filled with high- $n A_{M / I}=0.004$ nebulae and low- $n$ IB clouds. Relevant line ratios for each component, normalized by $\mathrm{H} \beta$, are listed in Table 3. The composite model represents a weighted sum of the three components, and a good overall match with the nucleus observations was obtained with relative $\mathrm{H} \beta$ contributions from (high- $n$ $\mathrm{MB}$ ): (high- $n$ IB): (low- $n$ IB) $=0.02: 0.68: 0.3$ (note that the high- $n$ MB : high- $n$ IB ratio is already fixed by $A_{M / I}$ ). The composite predicted ratios and observed values are listed in the last two columns of Table 3 .

While the 3-component simulation should not be taken too literally as a physical representation of the nebular structure in the M87 nucleus, the comparison presented here nonetheless provides an important demonstration that a multi-component photoionized plasma may account for the emission-line properties of this source. The choice of nebular parameters in this picture is not entirely arbitrary. Gas with densities ranging from $\sim 100 \mathrm{~cm}^{-3}$ to $\sim 10^{6} \mathrm{~cm}^{-3}$ exists in the cores of AGNs, and more general arguments exist for the importance of composite cloud populations in these environments. In particular, Baldwin et al. (1995) and Ferguson et al. (1997) have discussed the phenomenon of locally optimally emitting clouds (LOCs), in which emission in a particular line emerges predominantly from the subset of clouds with appropriately favorable nebular conditions for that transition. In the spirit of the LOC models, we conclude that it appears likely that a combination of high-density nebulae emit most of the lines observed in the nucleus of M87, while lower density clouds lead to the emission from ions with low critical densities, such as [O II] $\lambda 3727$ and [S II] $\lambda \lambda 6717,6731$. Analyses of other LINERs have similarly suggested that multiple cloud populations may be required to account for the observed emission-line properties (e.g., Péquignot 1984; Barth et al. 2001).

An interesting point which our high- $n A_{M / I}$ calculations raise is whether the UV line-ratio diagrams are in fact robust in distinguishing between shock heating and photoionization, if density effects are taken into consideration. Figures 2 and 3 show that the line ratios from the ionized disk 
of M87 could be well described by the high- $n A_{M / I}=0.006$, except for [O II $] \lambda 3727$. We again attempted to construct a composite model with different combinations of the high- $n A_{M / I}$ and low- $n$ IB components. We found that the same fractional contributions of the components used above (with $\mathrm{H} \beta$ proportions 0.7 : 0.3 ) give reasonably satisfactory results, although low- $n_{\text {crit }}$ lines such as [O II] and [S II] $\lambda \lambda 6717,6731$ remain underpredicted by a factor of $\sim 2$. We conclude that the extended disk of M87 remains a good candidate for shock ionization, but emphasize that the UV line diagnostics advocated by D97 and Allen et al. (1998) may encounter limitations when applied to composite nebular systems that include high-density components.

\subsection{ENERGETICS}

A consistency test for photoionization interpretations of the nuclear nebula in M87 is whether the central source provides sufficient ionizing photons to power the line emission. To address this point, we employed recent measurements of the nuclear continuum as measured by XMM-Newton in 2000 (Böhringer et al. 2001). These data have the advantage of simultaneously sampling the UV and X-ray bandpasses; while the central source is known to be variable, we employ these measurements as a representative snapshot of the broad-band continuum. The narrow lines in this source are neither known nor expected to be variable. In the UV bandpass, the XMM-Newton data provide a flux density for the M87 nucleus of $(1.90 \pm 0.04) \times 10^{-27} \mathrm{erg} \mathrm{s}^{-1} \mathrm{~cm}^{-2} \mathrm{~Hz}^{-1}$ at $2120 \AA$, and an integrated X-ray flux of $1.5 \times 10^{-12} \mathrm{erg} \mathrm{s}^{-1} \mathrm{~cm}^{-2}$ for $2-10 \mathrm{keV}$.

We can extrapolate the XMM-Newton UV flux by assuming that the spectral shape is the same as during our HST observation. We first attempted to derive an analytic fit to the continuum we measured for the nucleus, using SPECFIT as before. The continuum was represented by a broken power law subject to reddening, combined with a stellar component represented by the template described in $\S 2$. The fit indicates that the galaxy starlight contribution is negligible, and the continuum is well-represented by only a power law with a break at $4501 \pm 6 \AA$, subject to reddening given by $A_{V}=0.15 \pm 0.01 \mathrm{mag}$. This amount of reddening is reasonably consistent with the value of $0.11 \pm 0.02 \mathrm{mag}$ predicted from the Galactic H I column density toward M87 $\left[N_{H I}=(2.1 \pm 0.3) \times 10^{20} \mathrm{~cm}^{-2}\right.$; Sankrit et al. 1999] and a standard $N_{H I} / A_{V}$ ratio (Bohlin, Savage, $\&$ Drake 1978). The spectral indices of the power law are $\alpha=-1.35 \pm 0.02$ and $\alpha=-1.78 \pm 0.02$ for the red and blue portions, respectively. The spectrum of the nucleus along with this fit are shown in Figure 4. A similar fit to the FOS spectra was reported by Tsvetanov et al. (1999b). Our 1999 STIS flux at $2120 \AA$ is $(7.4 \pm 0.6) \times 10^{-28} \mathrm{erg} \mathrm{s}^{-1} \mathrm{~cm}^{-2} \mathrm{~Hz}^{-1}$, a factor of 2.6 below that obtained in 2000 by XMM-Newton, which provides one indicator of the level of variability between these two measurements. Scaling our continuum observation to bring it into agreement with the XMM-Newton data, and removing reddening corresponding to $A_{V}=0.15$ mag, implies a flux density at $2500 \AA$ of $3.6 \times 10^{-27} \mathrm{erg} \mathrm{s}^{-1} \mathrm{~cm}^{-2} \mathrm{~Hz}^{-1}$.

At higher energies, the continuum is constrained by the XMM-Newton X-ray measurements. Böhringer et al. (2001) reported that the $0.6-10 \mathrm{keV}$ spectrum was successfully described assuming 
Galactic absorption only, and a power law with best-fitting $\alpha=-1.2$. Using the same spectral index with the $2-10 \mathrm{keV}$ flux noted above implies that the $2 \mathrm{keV}$ flux density is $2.2 \times 10^{-30}$ erg $\mathrm{s}^{-1} \mathrm{~cm}^{-2} \mathrm{~Hz}^{-1}$. The two-point spectral index connecting $2500 \AA$ and $2 \mathrm{keV}$ is then $\alpha_{\text {ox }} \approx-1.2$. While this value is the same as that measured in X-rays, it is shallower than the measured UV index of -1.78 , implying that the spectrum must show some spectral curvature across the ionizing ultraviolet region. If we approximate the continuum with a single power law with $\alpha \approx-1.2$, normalized to match the dereddened $2500 \AA$ flux, the hydrogen-ionizing photon flux at the Earth in the absence of absorption would be 0.12 photons $\mathrm{s}^{-1} \mathrm{~cm}^{-2}$. After correction for $A_{V}=0.15$ mag, the $\mathrm{H} \beta$ photon flux in our STIS nucleus aperture is $1.90 \times 10^{-3}$ photons s $\mathrm{cm}^{-2}$, implying a photon ratio $Q_{i o n} / Q_{\mathrm{H} \beta}=63$. For comparison, Case B recombination at a temperature of $10^{4} \mathrm{~K}$ and density of $10^{3} \mathrm{~cm}^{-3}$ predicts $Q_{i o n} / Q_{\mathrm{H} \beta}=8.55$ (Hummer \& Storey 1987). It thus seems likely that the central source provides enough ionizing photons to power the emission nebula within the central $\sim 1^{\prime \prime}$, if the covering factor for the nebular gas is $\sim 10-20 \%$.

The nebular disk extends beyond the aperture employed here for the nucleus, however. The surface brightness distribution for the nebular disk has been studied by Ford et al. (1994), D97, and Tsvetanov et al. (1999a) using HST narrow-band imaging of $\mathrm{H} \alpha+[\mathrm{N} \mathrm{II}]$. Within $1^{\prime \prime}$ of the nucleus, the resulting integrated $\mathrm{H} \alpha+[\mathrm{N} \mathrm{II}]$ flux is $(2.0 \pm 0.7) \times 10^{-13} \mathrm{erg} \mathrm{cm}^{-2} \mathrm{~s}^{-1}$ (Ford et al. 1994). If we integrate out from this radius the analytic fit provided by Tsvetanov et al. (1999a) for the surface brightness distribution, the total extrapolated $\mathrm{H} \alpha+[\mathrm{N} \mathrm{II}]$ flux for the disk is then $2.6 \times 10^{-13} \mathrm{erg} \mathrm{s}^{-1} \mathrm{~cm}^{-2}$. This flux exceeds our nuclear measurement by a factor of $\sim 3.3$; if we scale our $\mathrm{H} \beta$ flux by a corresponding amount, the ratio $Q_{i o n} / Q_{\mathrm{H} \beta} \approx 19$, which still allows consistency with photoionization if the covering factor is at least $\sim 40-50 \%$.

The calculation described above for the ionizing photon flux is uncertain in several ways. First, it requires an uncertain extrapolation across the unobservable ionizing UV bandpass that contains most of $Q_{i o n}$. In addition, the continuum source is variable. It is therefore uncertain whether our choice of flux level adopted for this calculation is close to an average value. The calculation above involves a further assumption that the continuum we measure is emitted isotropically at the source. If a portion of this continuum is in fact beamed in our direction, our estimate of the photon production available for photoionizing the nebula would be overestimated. Some amount of beaming would not be surprising in light of other similarities between the M87 nucleus and BL Lac objects (see Tsvetanov et al. 1998 for discussion). In any case, it appears likely that the energetics change from being driven primarily by the nuclear radiation field, to other processes at larger radii; on large scales $\left(\lesssim 10^{\prime \prime}\right)$, the total integrated line flux grows to $1.2 \times 10^{-12} \mathrm{erg} \mathrm{s}^{-1}$ $\mathrm{cm}^{-2}$, implying as above $Q_{i o n} / Q_{\mathrm{H} \beta} \approx 4$, which is likely to be problematic. Taken at face value, the models for photoionization of the nucleus (§3.1) and shock heating of the disk (D97) suggest that this transition occurs within only a few tens of parsecs from the center. 


\subsection{AbSorption Lines}

The absorption lines detected in our spectra provide another diagnostic of material that may be in close proximity to the AGN. The data with the best combination of (high) spectral resolution and signal-to-noise ratio, from STIS G140L and FOS G270H, can be used to estimate some of the properties of the absorbing matter. Absorption lines identified in these spectra and measurements of their equivalent widths (EWs) are presented in Table 4, where we have also included measurements of the $\mathrm{Ca}$ II $\mathrm{K}+\mathrm{H}$ and $\mathrm{Na}$ I D lines from the FOS G400H and G570H spectra, respectively. The lines were measured using SPECFIT assuming Gaussian profiles. We show in Figure 5 close-up views of the spectra and fits around the absorption lines. Tsvetanov et al. (1999b) have reported the detection of absorption lines in the same FOS spectra that we are using.

The velocity of the absorption lines relative to that of the emission lines in M87 is of importance for determining the nature and physical state of the absorber. The measured wavelengths listed in Table 4 reflect the wavelength scale resulting from the pipeline reduction of the spectra, which may be subject to significant zero-point shifts. The average velocity of the absorption lines measured with the FOS G270H, not including lines blended with Galactic features, is $1091 \pm 11 \mathrm{~km} \mathrm{~s}^{-1}$; for the STIS G140L spectrum, the mean velocity is $1066 \pm 36 \mathrm{~km} \mathrm{~s}^{-1}$. These values are consistent within their uncertainties, and for each grating considered individually the velocities of the individual lines are likewise consistent with each other. We also fit emission lines in the same spectra. For FOS G270H, C II] $\lambda 2326$ appears at $2334.9 \pm 0.5 \AA$, corresponding to a velocity of $1073 \pm 66 \mathrm{~km} \mathrm{~s}^{-1}$; for STIS G140L, C IV $\lambda 1549$ appears at $1555.3 \pm 0.6 \AA$ and He II $\lambda 1640$ appears at $1647.0 \pm 0.6$ $\AA$, yielding velocities of $1220 \pm 120 \mathrm{~km} \mathrm{~s}^{-1}$ (assuming an optically thin C IV doublet ratio) and $1200 \pm 110 \mathrm{~km} \mathrm{~s}^{-1}$, respectively. These results indicate that the absorber and the emitter are at approximately the same redshift. The G140L measurement yields a wavelength for Ly $\alpha$ of $1221.45 \pm 0.06 \AA$, corresponding to $1426 \pm 15 \mathrm{~km} \mathrm{~s}^{-1}$, which is significantly larger than the results for the other lines; however, this finding is consistent with expectations that the Ly $\alpha$ emission may be modified significantly (30\% - 40\% flux reduction) due to absorption by Galactic H I, resulting in a redward shift of the transmitted line centroid (Sankrit et al. 1999).

We can improve estimates of absolute velocity from these spectra by associating the Galactic absorption features with H I $21 \mathrm{~cm}$ absorption measured toward M87. Results from Davis \& Cummings (1975) indicate that radio absorption arising in the Milky Way along this sightline is spread over a $50 \mathrm{~km} \mathrm{~s}^{-1}$ range, with the major component at $\sim-8 \mathrm{~km} \mathrm{~s}^{-1}$ with respect to the Local Standard of Rest; this velocity corresponds to a heliocentric value of $\sim-12 \mathrm{~km} \mathrm{~s}^{-1}$. The average redshift of Galactic lines in the FOS G270H spectrum is nominally $-72 \pm 16 \mathrm{~km} \mathrm{~s}^{-1}$, implying that a correction of $+60 \pm 16 \mathrm{~km} \mathrm{~s}^{-1}$ should be added to velocities derived from this spectrum in order to obtain heliocentric values. The heliocentric velocity of the M87 absorber is then $1151 \pm 19$ $\mathrm{km} \mathrm{s}^{-1}$, based on the $\mathrm{G} 270 \mathrm{H}$ measurement. This result means that the absorber is blueshifted by $\sim 126 \pm 19 \mathrm{~km} \mathrm{~s}^{-1}$ with respect to M87, which is at $1277 \pm 2 \mathrm{~km} \mathrm{~s}^{-1}$ as determined from stellar absorption lines (van der Marel 1994). The data for C II] in the G270H spectrum likewise implies that the emission-line plasma is also blueshifted with respect to the host galaxy, by $144 \pm 68 \mathrm{~km} \mathrm{~s}^{-1}$. 
We carried out a curve-of-growth analysis to calculate the column densities of the absorbing ions. We followed the procedure outlined in Spitzer (1978) using our observed equivalent widths. One major uncertainty in this derivation is the detailed shape of the absorption features, since the lines that we detect are not entirely resolved; some substructure within the lines is suggested from previous observations of the Na I D lines (Carter, Johnstone, \& Fabian 1997). We consequently chose a Doppler parameter $b=\mathrm{FWHM} / 1.665=200 \mathrm{~km} \mathrm{~s}^{-1}$ for consistency with the intrinsic FWHM of the lines as measured in the FOS and STIS spectra. We used the oscillator strengths from Verner et al. (1994). The resulting values of the column densities $N_{i o n}$ are listed in Tables 4 and 5. Given the low spectral resolution and possibility of unresolved substructure in the lines, the resulting $N_{i o n}$ estimates should be treated as lower limits. This point is reinforced by the tendency for some ions to display lower calculated column densities for lines with larger oscillator strengths, consistent with the presence of saturated substructure within these lines.

The results for the individual ions can be used to place constraints on the total column density of the absorber. Each $N_{\text {ion }}$ gives a lower limit to the corresponding $N_{\text {element }}$, which in turn provides a lower limit to the total hydrogen column density $N_{H}$, assuming solar abundances (Anders \& Grevesse 1989). The inferred $N_{H}$ from the various ions, whose absorption lines are not contaminated by absorption from other absorption features, indicates that $N_{H} \gtrsim 10^{18} \mathrm{~cm}^{-2}$, except from the column densities of Ca II $\left(N_{H} \gtrsim 10^{19} \mathrm{~cm}^{-2}\right)$ and Mn II $\left(N_{H} \gtrsim 10^{20} \mathrm{~cm}^{-2}\right)$.

The total $N_{H}$ in the M87 absorber can be constrained by other means. Dwarakanath, van Gorkom, \& Owen (1994) used H I $21 \mathrm{~cm}$ observations to place an upper limit on the absorbing H I column density of $5 \times 10^{19} \mathrm{~cm}^{-2}$. (This value is sensitive to the spin temperature, assumed by these authors to be $100 \mathrm{~K}$.) Since the absorption lines detected in the HST spectra arise only from very low ionization species (requiring ionization energies $<13.6 \mathrm{eV}$ to produce), the hydrogen in the absorber is expected to be mostly neutral, and this value can thus be taken as a useful limit on the total $N_{H}$. As noted in $\S 3.2$ for the nucleus, the reddening of the optical/UV continuum and the Xray absorbing column as measured by XMM-Newton both appear to be dominated by the Galactic contribution, suggesting that the absorber associated with M87 has $N_{H} \lesssim 10^{20} \mathrm{~cm}^{-2}$. Recent Chandra X-ray measurements reported by Di Matteo et al. (2002) indicate that any absorbing matter associated with the nucleus has $N_{H}<3.2 \times 10^{20} \mathrm{~cm}^{-2}$. A consistent picture thus emerges in which the absorber has $N_{H} \approx 10^{19}-10^{20} \mathrm{~cm}^{-2}$. The only potential disagreement arises from the lower limit of $N_{H} \gtrsim 10^{20} \mathrm{~cm}^{-2}$ obtained above from the Mn II absorption lines. The Mn II $\lambda 2577$ line is potentially contaminated by Galactic absorption in Fe II $\lambda 2587$, and Mn II $\lambda 2594$ suffers a similar problem with Galactic Mn II $\lambda 2606$, leaving the Mn II $\lambda 2606$ in M87 as the best option for measuring this ion; the measurement uncertainty for this feature is relatively large, however $(1 \sigma$ error of $26 \%$; see also Fig. $5 d$ ), suggesting that it does not represent a significant inconsistency.

A fundamental question concerning the absorber in M87 is its relation, if any, to the accretionpowered activity in the nucleus. LINERs commonly exhibit resonance-line absorption tracing modest column densities, and these absorbers characteristically display a low-ionization state that mirrors the low ionization of the emission plasma in these sources (Shields et al. 2002, and references 
therein). These absorbers are not particularly exotic in their properties, and a natural question is whether they have any connection to the active nucleus, or simply represent normal components of the interstellar medium of the host galaxy that happen to fall along our line of sight to the central UV source. The latter possibility is underscored by the similarity in detected ions and equivalent widths for the Galactic and M87 absorbers (Tables 4 and 5).

While the nature of absorbers in LINERs in general remains ambiguous, in the case of M87 there are indications that the absorbing material is, in fact, associated with the central accretion phenomenon. The offset in velocity between the absorber and the underlying galaxy, indicative of outflow of the absorbing matter, is highly reminiscent of absorption commonly associated with more luminous AGNs, which is often blueshifted with typical velocities of a few $\times 100 \mathrm{~km} \mathrm{~s}^{-1}$ (e.g, Crenshaw et al. 1999). In addition, Sankrit et al. (1999) concluded that the substantial strength of the Ly $\alpha$ emission feature requires that the absorber cover the continuum source, but little if any of the emission-line clouds, if $N_{H} \gtrsim 10^{18} \mathrm{~cm}^{-2}$, as seems to be the case. The most straightforward way of interpreting this finding geometrically is that the absorber has a distance from the central source and a size that are smaller than the typical dimensions of the emission clouds. Since the HST aperture employed by Sankrit et al. had a diameter of $0^{\prime \prime} 26 \approx 20 \mathrm{pc}$, the size and scale of the absorber are probably much smaller than this dimension, which would be consistent with an origin in the central accretion structure or its environs.

The absorption characteristics of M87 and possibly other LINERs may lead to important insights into the accretion process in these objects. The low ionization describing both absorption and emission sets these sources apart from Seyfert nuclei and QSOs, and likely arises from a characteristically different mode of accretion at low accretion rates. In particular, physical scenarios employing advection-dominated accretion flows (ADAFs), adiabatic inflow-outflow solutions (ADIOS), or other low radiative efficiency accretion structures are showing promise for interpreting these sources (see Quataert 2001 for a recent review). Substantial outflows of hot gas are predicted in some versions of these models (e.g., Blandford \& Begelman 1999; Beckert 2000), and potentially some cooler material is accelerated in these winds, giving rise to the observed absorption. One quantity of interest for comparison with theoretical predictions is the rate of mass ejection $\dot{M}$ represented by the M87 absorber. From simple geometrical considerations, $\dot{M} \approx \mu m_{p} f N_{H} 4 \pi r v$, where $\mu$ is the mean atomic mass per $\mathrm{H}$ atom, $m_{p}$ is the proton mass, $f$ is the global covering factor, $r$ is the radial location of the absorber, and $v$ is the outflow velocity. Assuming solar abundances ( $\mu=1.41$; Däppen 2000), this can be expressed as

$$
\dot{M} \approx 0.002 f\left(\frac{N_{H}}{10^{20} \mathrm{~cm}^{-2}}\right)\left(\frac{r}{1 \mathrm{pc}}\right)\left(\frac{v}{126 \mathrm{~km} \mathrm{~s}^{-1}}\right) \mathrm{M}_{\odot} \mathrm{yr}^{-1} .
$$

This result indicates that $\dot{M}$ is much less than the Bondi accretion rate for the central object, which is $\sim 0.1 \mathrm{M}_{\odot} \mathrm{yr}^{-1}$ (Di Matteo et al. 2002), but consistent with the idea that the absorbed gas may be material entrained in an outflow from the central accretion structure. Observations with higher spectral resolution will be necessary to establish the detailed kinematic and spatial structure of the absorbing gas in M87 and other LINERs. 


\section{Conclusions}

The present study of UV/optical emission and absorption in the nearby LINER M87 provides several new insights into the nature of this source. Within a radius of $\sim 20 \mathrm{pc}$, emission-line ratios for the nucleus are well-described by photoionization of a multi-phase medium with a range of densities; shock models are less successful. The data also show a low-ionization absorber that

evidently resides on small scales and is outflowing. These attributes, along with the famous radio jet, strongly resemble those of more luminous AGNs, and clearly point to accretion as the underlying power source responsible for the LINER behavior.

M87 is, however, a good case for a composite system in terms of the ionization process generating the optical LINER. The emission-line characteristics of the resolved nebula change significantly away from the center; the emission-line ratios (D97) as well as energetics arguments suggest that mechanical heating becomes important for this gas on scales of tens of parsecs. If this pattern is typical, larger apertures common in ground-based measurements will sample emission plasma that may trace a complex mix of energetic processes. Efforts to identify a single, or even dominant, ionization mechanism in these systems may thus be unproductive, although the question of whether alternatives to accretion power are important provides a good reason to conduct detailed investigations of other LINER galaxies that lack broad lines or other clear signatures of accretion.

Support for this research was provided by NASA through grants GO-07357 and GO-08607 from the Space Telescope Science Institute, which is operated by the Association of Universities for Research in Astronomy, Inc., under NASA contract NAS5-26555. We also acknowledge NASA grant NAG5-3556 to A.V.F. We thank Gary Ferland for access to the photoionization code CLOUDY, Matthias Dietrich and Fred Hamann for helpful discussions, and the anonymous referee for constructive comments.

\section{REFERENCES}

Allen, M. G., Dopita, M. A., \& Tsvetanov, Z. I. 1998, ApJ, 493, 571

Anders, E., \& Grevesse, N. 1989, Geochim. Cosmochim. Acta, 53, 197

Baldwin, J., Ferland, G., Korista, K., \& Verner, D. 1995, ApJ, 455, L119

Barth, A. J., Ho, L. C., Filippenko, A. V., Rix, H.-W., \& Sargent, W. L. W. 2001, ApJ, 546, 205

Barth, A. J., Reichert, G. A., Filippenko, A. V., Ho, L. C., Shields, J. C., Mushotzky, R. F., \& Puchnarewicz, E. M. 1996, AJ, 112, 1829

Barth, A. J., Reichert, G. A., Ho, L. C., Shields, J. C., Filippenko, A. V., \& Puchnarewicz, E. M. 1997, AJ, 114, 2313

Beckert, T. 2000, ApJ, 539, 223 
Bicknell, G. F., \& Begelman, M. C. 1996, ApJ, 467, 597

Binette, L., Wilson, A. S., \& Storchi-Bergmann, T. 1996, A\&A, 312, 365

Blandford, R. D., \& Begelman, M. C. 1999, MNRAS, 303, L1

Bohlin, R. C., Savage, B. D., \& Drake, J. F. 1978, ApJ, 224, 132

Boksenberg, A., et al. 1992, A\&A, 261, 393

Cardelli, J. A., Clayton, G. C., \& Mathis, J. S. 1989, ApJ, 345, 245

Carter, D., Johnstone, R. M., \& Fabian, A. C. 1997, MNRAS, 285, L20

Crenshaw, D. M., Kraemer, S. B., Boggess, A., Maran, S. P., Mushotzky, R. F., \& Wu, C.-C. 1999, ApJ, 516, 750

Däppen, W. 2000, in Allen's Astrophysical Quantities, 4th ed., ed. A. N. Cox (New York: Springer), 29

Davies, R. D., \& Cummings, E. R. 1975, MNRAS, 170, 95

Di Matteo, T., Allen, S. W., Fabian, A. C., Wilson, A. S., \& Young, A. J. 2002, ApJ, in press (astro-ph/0202238v3)

Dopita, M. A., \& Sutherland, R. S. 1995, ApJS, 102, 161

Dopita, M. A., et al. 1997, ApJ, 490, 202 (D97)

Dwarakanath, K. S., van Gorkom, J. H., \& Owen, F. N. 1994, ApJ, 432, 469

Ferguson, J. W., Korista, K. T., Baldwin, J. A., \& Ferland, G. J. 1997, ApJ, 487, 122

Ferland, G. J., Korista, K. T., Verner, D. A., Ferguson, J. W., Kingdon, J. B., \& Verner, E. M. 1998, PASP, 749, 761

Filippenko, A. V. 1985, ApJ, 289, 475

Filippenko, A. V. 1996, in ASP Conf. Ser. 103, The Physics of LINERs, ed. M. Eracleous et al. (San Francisco: ASP), 17

Filippenko, A. V., \& Halpern, J. P. 1984, ApJ, 285, 458

Ford, H. C., et al. 1994, ApJ, 435, 27

Harms, R. J., et al. 1994, ApJ, 435, 35

Heckman, T. M. 1980, A\&A, 87, 152

Ho, L. C., Filippenko, A. V., \& Sargent, W. L. W. 1997, ApJ, 487, 568

Hummer, D. G., \& Storey, P. J. 1987, MNRAS, 224, 801

Kriss, G. 1994, in ASP Conf. Ser. 61, Astronomical Data Analysis Software and Systems III, ed. D. R. Crabtree, R. J. Hanisch, \& J. Barnes (San Francisco: ASP), 437

Laor, A. 1998, ApJ, 496, L71

Macchetto, F., Marconi, A., Axon, D. J., Capetti, A., Sparks, W., \& Crane, P. 1997, ApJ, 489, 579 
Maoz, D., Koratkar, A., Shields, J. C., Ho, L. C., Filippenko, A. V., \& Sternberg, A. 1998, AJ, 116,55

Nicholson, K. L., Reichert, G. A., Mason, K. O., Puchnarewicz, E. M., Ho, L. C., Shields, J. C., \& Filippenko, A. V. 1998, MNRAS, 300, 893

Osterbrock, D. E. 1989, Astrophysics of Gaseous Nebulae and Active Galactic Nuclei (Mill Valley, CA: University Science Books)

Pelat, D., Alloin, D., \& Fosbury, R. A. E. 1981, MNRAS, 195, 787

Péquignot, D. 1984, A\&A, 131, 159

Quataert, E. 2001, in ASP Conf. Ser. 224, Probing the Physics of Active Galaxies by Multiwavelength Monitoring, ed. B. M. Peterson, R. S. Polidan, \& R. W. Pogge (San Francisco: ASP), 71

Reynolds, C. S., Di Matteo, T., Fabian, A. C., Hwang, U., \& Canizares, C. R. 1996, MNRAS, L111

Sankrit, R., Sembach, K. R., \& Canizares, C. R. 1999, ApJ, 527, 733

Schlegel, D. J., Finkbeiner, D. P., \& Davis, M. 1998, ApJ, 500, 525

Shields, J. C., Sabra, B. M., Ho, L. C., Barth, A. J., \& Filippenko, A. V. 2002, in ASP Conf. Ser. 255, Mass Outflow in Active Galactic Nuclei: New Perspectives, ed. D. M. Crenshaw, S. B. Kraemer, \& I. M. George (San Francisco: ASP), 105

Spitzer, L., Jr. 1978, Physical Processes in the Interstellar Medium (New York: Wiley)

Tonry, J. L., Dressler, A., Blakeslee, J. P., Ajhar, E. A., Fletcher, A. B., Luppino, G. A., Metzger, M. R. \& Moore, C. B. 2001, ApJ, 546, 681

Tsvetanov, Z. I., Allen, M. G., Ford, H. C., \& Harms, R. J. 1999a, in the Radio Galaxy Messier 87, ed. H.-J. Röser \& K. Meisenheimer (Berlin: Springer), 301

Tsvetanov, Z. I., Hartig, G. E., Ford, H. C., Dopita, M. A., Kriss, G. A., Pei, Y. C., Dressel, L. L., \& Harms, R. J. 1998, ApJ, 493, L83

Tsvetanov, Z. I., Hartig, G. E., Ford, H. C., Kriss, G. A., Dopita, M. A., Dressel, L. L., \& Harms, R. J. 1999b, in the Radio Galaxy Messier 87, ed. H.-J. Röser \& K. Meisenheimer (Berlin: Springer), 307

van der Marel, R. P. 1994, MNRAS, 270, 271

Verner, D. A., Barthel, P. D., \& Tytler, D. 1994, ApJS, 108, 287

Whysong, D., \& Antonucci, R. 2002, astro-ph/0207385v1

This preprint was prepared with the AAS LATEX macros v5.0. 

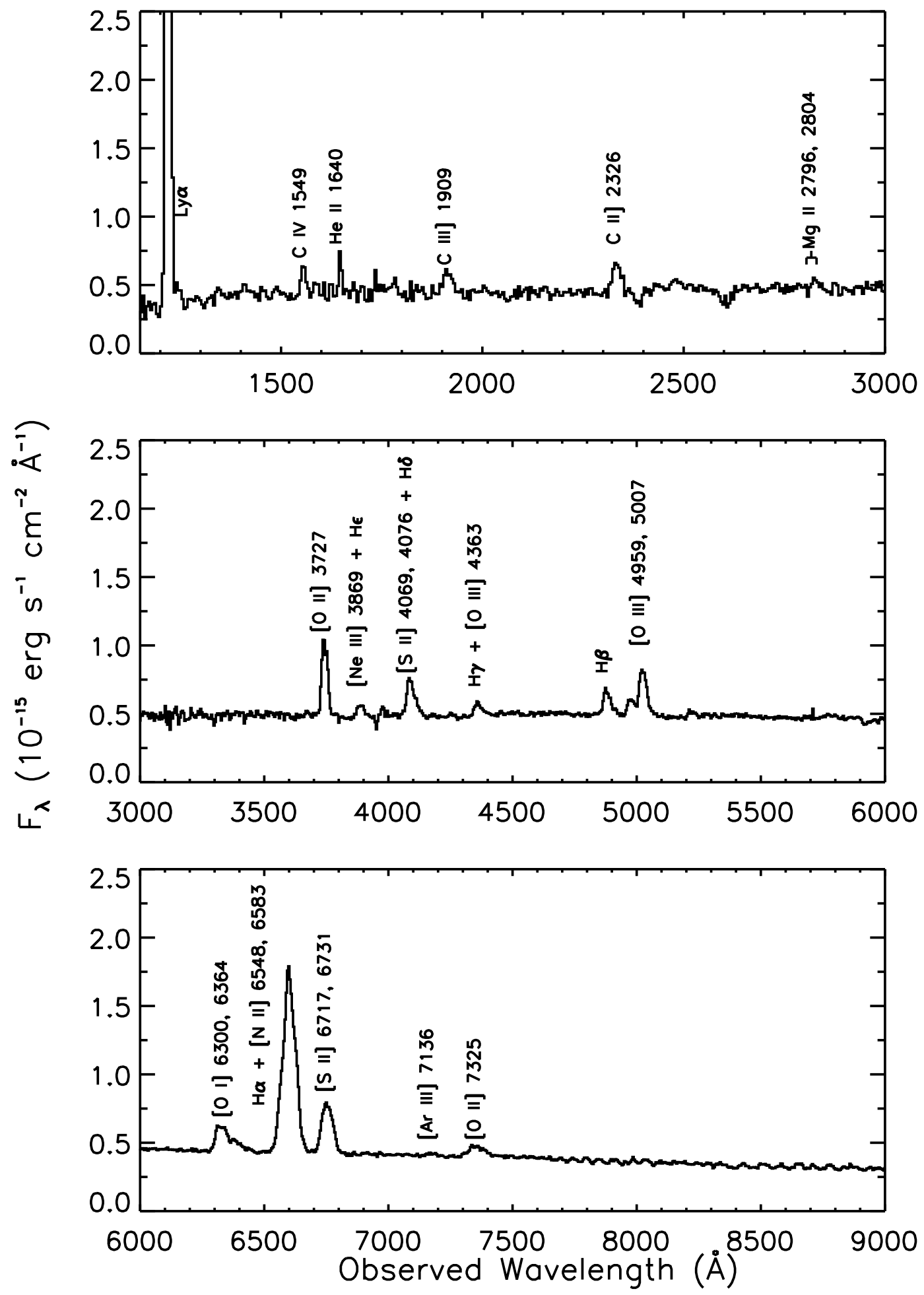

Fig. 1. - STIS spectrum of the M87 nucleus. Undulations in the continuum redward of $\sim 7700 \AA$ are artifacts of fringing in the CCD detector. 

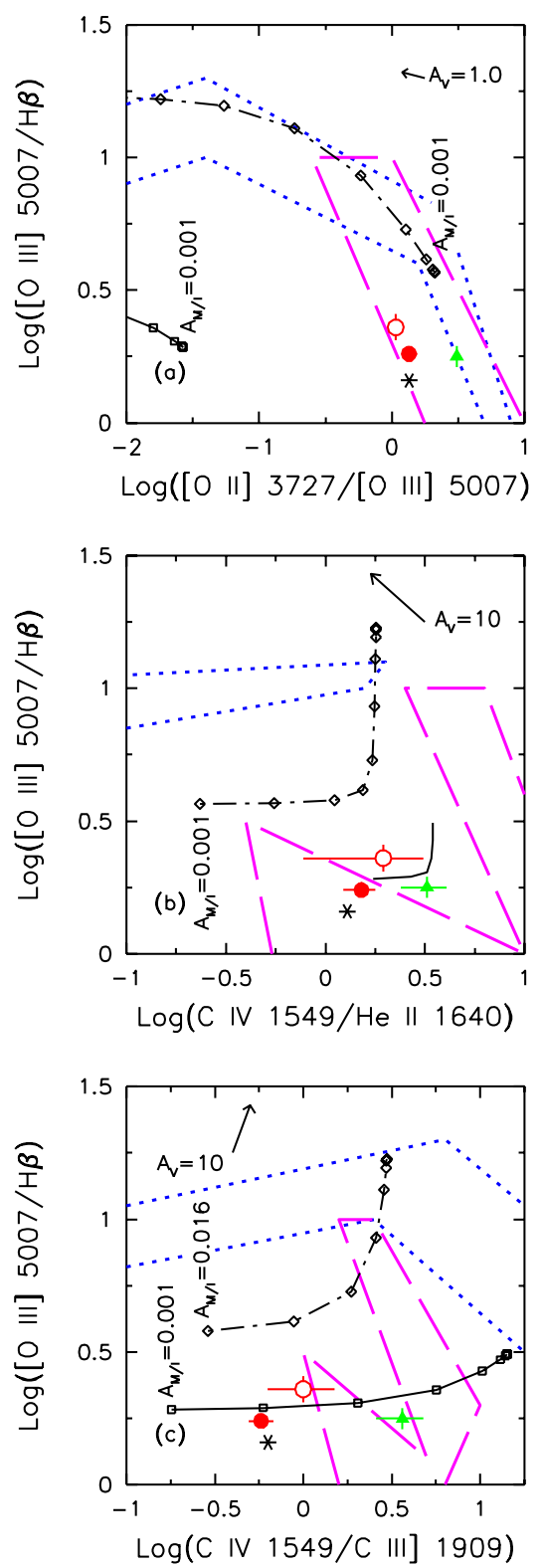

Fig. 2.- Optical and UV line ratio diagrams. Our measurements for the M87 nucleus are represented by filled (STIS) and open (FOS) red circles, while green triangles represent measurements on the ionized disk (D97). Blue dotted lines show the loci of photoionization predictions, while magenta dashed lines indicate the ratios from the Dopita \& Sutherland (1995) shock models. The low-density $A_{M / I}$ sequence with parameters from Binette et al. (1996) is shown by the black dashdot line with diamonds, while the high-density $A_{M / I}$ predictions described in $\S 3.1$ are represented by the black solid line with squares. (The square points for the models are suppressed in $(b)$ for the sake of clarity.) For both $A_{M / I}$ sequences, successive points represent increases of $A_{M / I}$ by a factor of two; the smallest value is indicated. The asterisk indicates the composite model predictions for high-density $A_{M / I}=0.004+$ low-density IB. The effect of extinction $\left(A_{V}=1.0\right.$ or $\left.10 \mathrm{mag}\right)$ is shown by the arrow. 

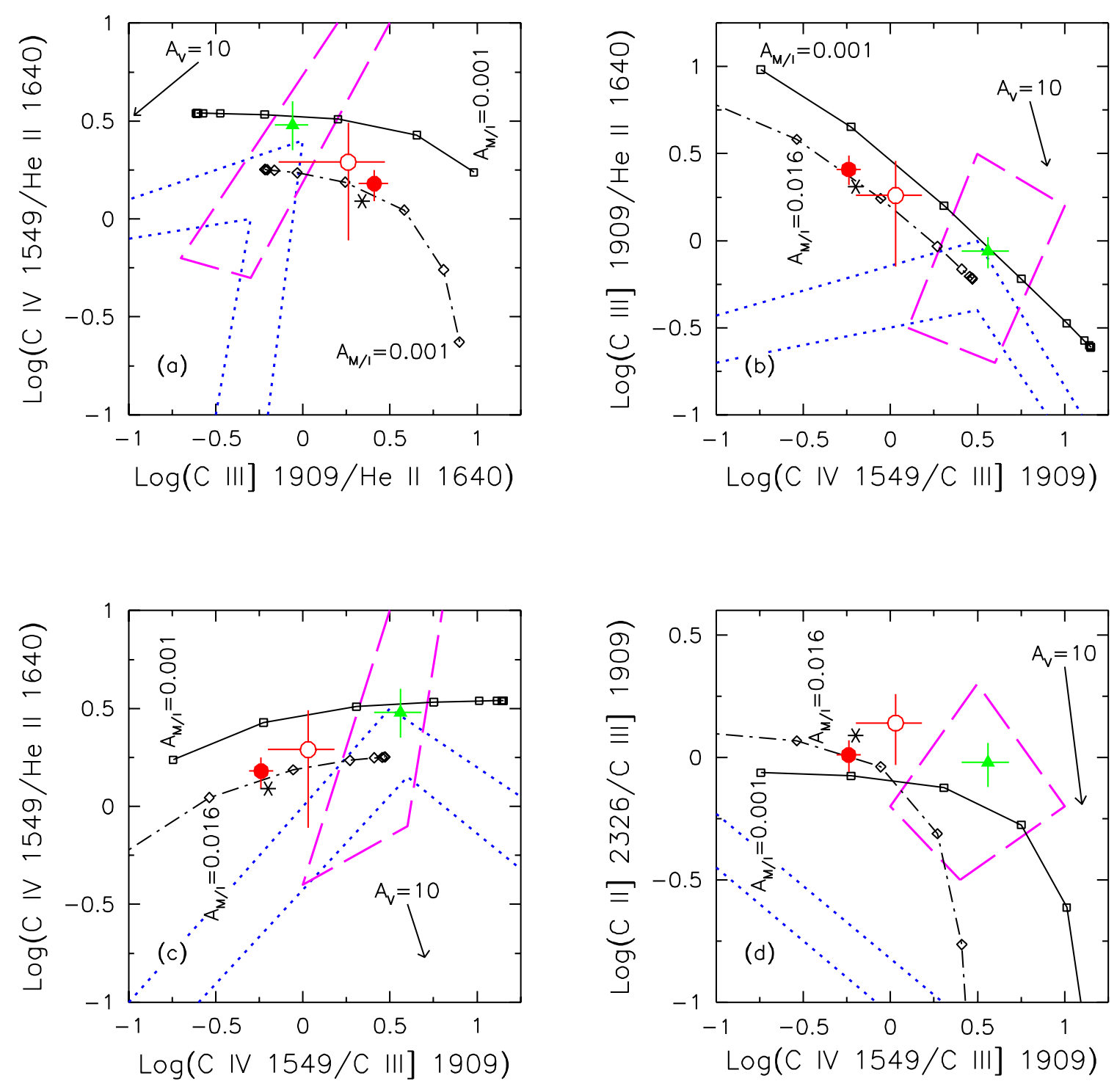

Fig. 3.- Same as for Figure 2, but for purely UV lines. 


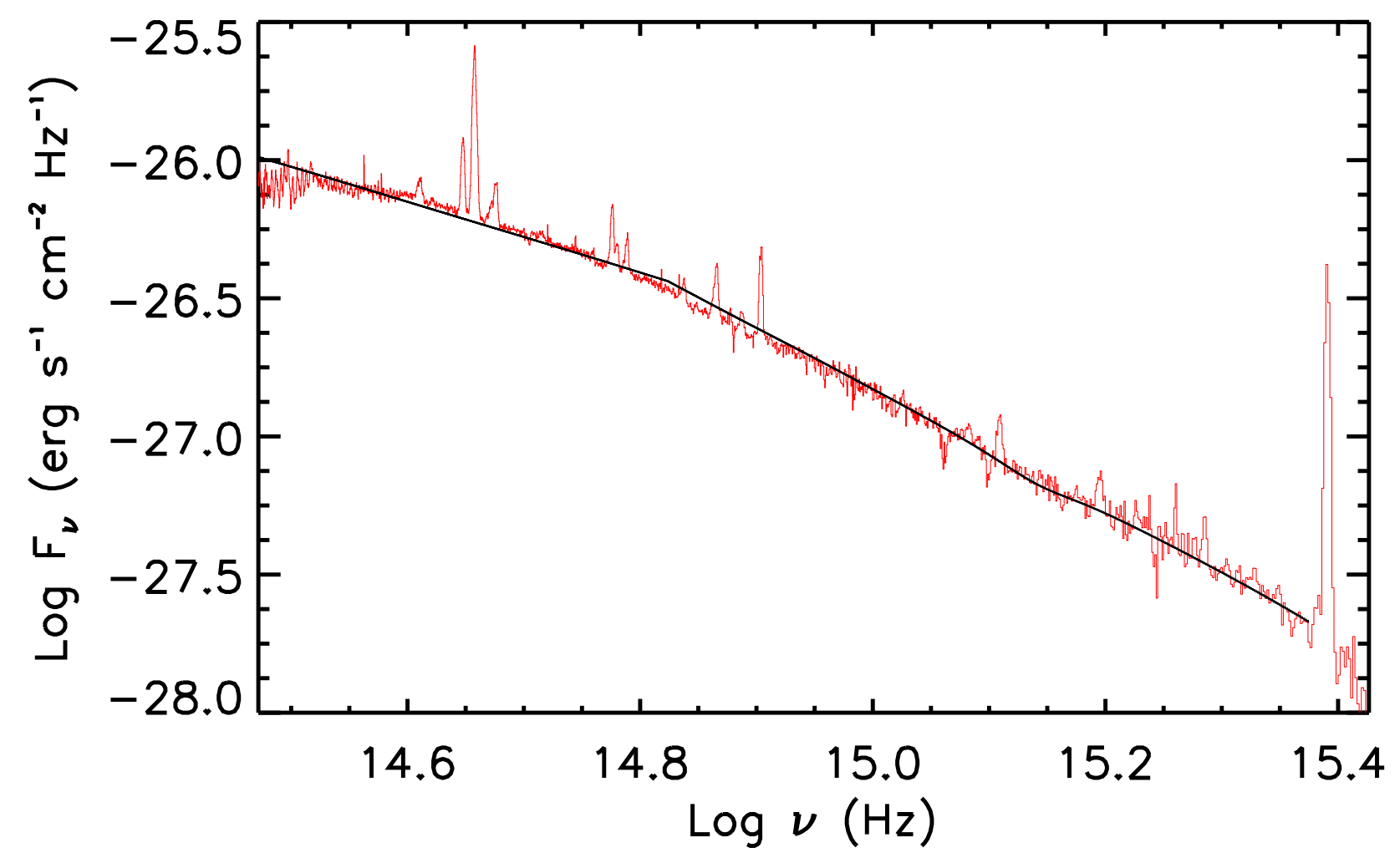

Fig. 4.- M87 nucleus spectrum (red line) and our model continuum fit (black line), consisting of a broken power-law modified by reddening. 

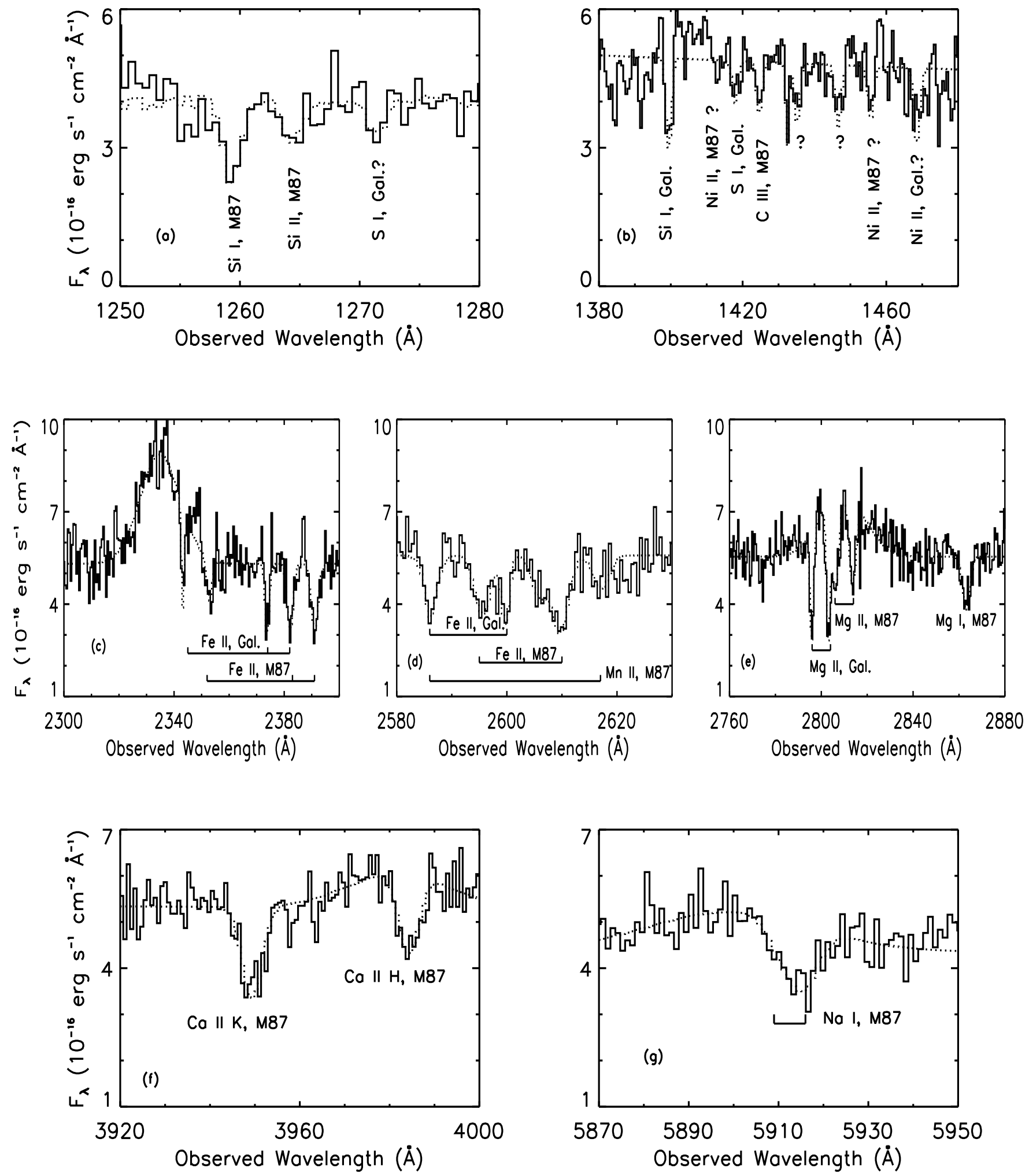

Fig. 5.- Absorption features in the FOS and STIS spectra of M87 (solid histogram) and our model fits (dotted lines). 
Table 1: HST STIS and FOS Observations

\begin{tabular}{cccccc}
\hline \hline STIS & & \multicolumn{4}{c}{ Spectral Resolution ${ }^{1}$} \\
Grating & Detector & Coverage $(\AA)$ & Point Source & Extended & Exp. (seconds) \\
\hline G140L & FUV-MAMA & $1150-1720$ & 190 & 2500 & 2590 \\
G230L & NUV-MAMA & $1590-3160$ & 600 & 4000 & 2296 \\
G430L & CCD & $2900-5710$ & 380 & 1900 & 900 \\
G750L & CCD & $5270-10270$ & 390 & 1900 & 1000 \\
\hline FOS & & & & & \\
Grating & Detector & Coverage $(\AA)$ & Point Source & Extended & Exp. (seconds) \\
\hline G160L & FOS/BL & $1140-2510$ & 1300 & 1300 & 2300 \\
G270H & FOS/RD & $2220-3275$ & 230 & 230 & 2300 \\
G400H & FOS/RD & $3235-4780$ & 230 & 230 & 2300 \\
G570H & FOS/RD & $4560-6820$ & 230 & 230 & 2300 \\
\hline
\end{tabular}

${ }^{1}$ FWHM resolution in $\mathrm{km} \mathrm{s}^{-1}$. 
Table 2: Observed Emission-Line Fluxes (STIS \& FOS)

\begin{tabular}{|c|c|c|}
\hline Emission Line & STIS Flux $^{1}$ & "FOS Flux ${ }^{2}$ \\
\hline $\operatorname{Ly} \alpha$ & $14.07 \pm 0.59$ & $30.86 \pm 2.96$ \\
\hline C IV $\lambda 1549$ & $0.60 \pm 0.06$ & $0.99 \pm 0.28$ \\
\hline He II $\lambda 1640$ & $0.40 \pm 0.06$ & $0.51 \pm 0.27$ \\
\hline C III] $\lambda 1909$ & $1.03 \pm 0.13$ & $0.92 \pm 0.27$ \\
\hline C II] $\lambda 2326$ & $1.05 \pm 0.08$ & $1.26 \pm 0.17$ \\
\hline Mg II $\lambda \lambda 2796,2804$ & $1.12 \pm 0.09$ & $1.24 \pm 0.17$ \\
\hline [O II] $\lambda 3727$ & $2.47 \pm 0.11$ & $2.45 \pm 0.18$ \\
\hline$[\mathrm{Ne}$ III $] \lambda 3869$ & $0.36 \pm 0.04$ & $0.79 \pm 0.09$ \\
\hline$[\mathrm{S} \mathrm{II}] \lambda 4072^{3}$ & $1.52 \pm 0.08$ & $2.22 \pm 0.19$ \\
\hline $\mathrm{H} \gamma^{4}$ & $0.49 \pm 0.04$ & $0.52 \pm 0.08$ \\
\hline $\mathrm{H} \beta$ & $1.00 \pm 0.05$ & $1.00 \pm 0.10$ \\
\hline [O III] $\lambda 4959$ & $0.61 \pm 0.03$ & $0.76 \pm 0.06$ \\
\hline [O III] $\lambda 5007$ & $1.84 \pm 0.08$ & $2.29 \pm 0.18$ \\
\hline$[\mathrm{O}$ I] $\lambda 6300$ & $1.62 \pm 0.08$ & $2.28 \pm 0.19^{5}$ \\
\hline [O I] $\lambda 6364$ & $0.53 \pm 0.03$ & \\
\hline $\mathrm{H} \alpha,[\mathrm{N} \mathrm{II}] \lambda 6548,83$ & $12.33 \pm 0.49$ & $13.29 \pm 0.95$ \\
\hline$[\mathrm{S}$ II $] \lambda 6717,6731$ & $3.10 \pm 0.13$ & $2.32 \pm 0.21$ \\
\hline$[\mathrm{Ar}$ III $] \lambda 7165^{6}$ & $0.10 \pm 0.04$ & \\
\hline$[\mathrm{O}$ II] $\lambda 7325$ & $0.87 \pm 0.08$ & \\
\hline
\end{tabular}

${ }^{1}$ Normalized to $\mathrm{H} \beta=6.45 \times 10^{-15} \mathrm{erg} \mathrm{s}^{-1} \mathrm{~cm}^{-2}$, uncorrected for reddening.

2 Normalized to $\mathrm{H} \beta=4.03 \times 10^{-15} \mathrm{erg} \mathrm{s}^{-1} \mathrm{~cm}^{-2}$, uncorrected for reddening.

${ }^{3}$ Blended with $\mathrm{H} \delta$.

${ }^{4}$ Blended with [O III] $\lambda 4363$.

${ }^{5}$ Includes [O I] $\lambda 6364$.

${ }^{6}$ Uncertain. 
Table 3: Photoionization Predictions ${ }^{1}$

\begin{tabular}{ccccccc}
\hline \hline Line & high- $n$ MB & high- $n$ IB & $A_{M / I}=0.004$ & low- $n$ IB & Total $^{2}$ & Observed $^{3}$ \\
\hline C IV $\lambda 1549$ & 26.76 & 0.05 & 0.76 & 0.00 & 0.53 & 0.60 \\
He II $\lambda 1640$ & 7.70 & 0.08 & 0.28 & 0.70 & 0.41 & 0.40 \\
C III] $\lambda 1909$ & 1.88 & 1.26 & 1.28 & 0.10 & 0.93 & 1.03 \\
C II] $\lambda 2326$ & 0.00 & 1.11 & 1.08 & 0.66 & 0.95 & 1.05 \\
{$[\mathrm{O} \mathrm{II}] \lambda 3727$} & 0.00 & 0.05 & 0.05 & 6.48 & 1.98 & 2.47 \\
{$[\mathrm{Ne}$ III $] \lambda 3869$} & 0.41 & 1.82 & 1.78 & 0.96 & 1.53 & 0.36 \\
{$[\mathrm{O} \mathrm{III}] \lambda 5007$} & 3.11 & 1.91 & 1.95 & 0.32 & 1.46 & 1.84 \\
{$[\mathrm{O} \mathrm{I}] \lambda 6300$} & 0.00 & 2.33 & 2.27 & 1.47 & 2.03 & 1.62 \\
$\mathrm{H} \alpha+\mathrm{N} \mathrm{II}] \lambda \lambda 6548,6584$ & 0.00 & 3.80 & 3.78 & 9.81 & 5.59 & 12.33 \\
{$[\mathrm{~S} \mathrm{II}] \lambda 6717,6731$} & 0.00 & 0.26 & 0.25 & 6.91 & 2.25 & 2.32 \\
\hline
\end{tabular}

1 All fluxes expressed relative to $\mathrm{H} \beta$.

2 Fluxes predicted for the composite, 3-component photoionization model.

3 STIS measurements, uncorrected for reddening.

Table 4: Absorption Lines (STIS, G140L)

\begin{tabular}{ccccc}
\hline \hline Line ID & $\lambda_{\text {obs }}(\AA)$ & $\mathrm{EW}(\AA)$ & $\log \left(\mathrm{N} \mathrm{cm}^{-2}\right)$ & Origin \\
\hline Si I $\lambda 1255^{1}$ & $1259.31 \pm 0.16$ & $0.75 \pm 0.10$ & 14.35 & M87 \\
Si II $\lambda 1260^{2}$ & $1264.49 \pm 0.33$ & $0.40 \pm 0.03$ & 12.42 & M87 \\
S I $\lambda 1270^{3}$ & $1271.40 \pm 0.29$ & $0.33 \pm 0.12$ & 15.86 & Gal.(?) \\
S I $\lambda 1401^{3}$ & $1399.44 \pm 0.16$ & $0.79 \pm 0.10$ & 15.59 & Gal. \\
Ni II $\lambda 1413^{3}$ & $1417.96 \pm 0.38$ & $0.38 \pm 0.10$ & 15.55 & M87 \\
S I $\lambda 1425^{3}$ & $1424.64 \pm 0.36$ & $0.47 \pm 0.14$ & 14.38 & Gal. \\
$\ldots \ldots \ldots$. & $1432.49 \pm 0.15$ & $0.39 \pm 0.09$ & $\ldots \ldots$ & Unknown \\
$\ldots \ldots \ldots$. & $1435.13 \pm 0.41$ & $0.55 \pm 0.15$ & $\ldots \ldots$ & Unknown \\
$\ldots \ldots \ldots$. & $1446.64 \pm 0.65$ & $0.59 \pm 0.16$ & $\ldots \ldots$ & Unknown \\
Ni II $\lambda 1450^{4}$ & $1455.69 \pm 0.36$ & $0.50 \pm 0.16$ & 15.93 & M87 \\
Ni II $\lambda 1468^{5}$ & $1468.55 \pm 0.79$ & $0.73 \pm 0.18$ & 16.30 & Gal.(?) \\
Si II $\lambda 1527$ & $1531.62 \pm 0.26$ & $0.70 \pm 0.19$ & 14.47 & M87 \\
\hline
\end{tabular}

${ }^{1}$ Contributions from Galactic S I $\lambda 1259.52$ and Fe II $\lambda 1260.53$.

${ }^{2}$ Contributions from M87 S I $\lambda 1259.52$ and Fe II $\lambda 1260.53$.

3 Multiple components.

${ }^{4}$ Contributions from M87 Co II $\lambda 1448.01$ and Galactic Ni II $\lambda 1454.84$.

${ }^{5}$ Contributions from Galactic Co II $\lambda 1466.20$ and Ni II $\lambda 1467.26$. 
Table 5: Absorption Lines (FOS, G270H, G400H, G570H)

\begin{tabular}{ccccc}
\hline \hline Line ID & $\lambda_{\text {obs }}(\AA)$ & $\mathrm{EW}(\AA)$ & $\log \left(\mathrm{N} \mathrm{cm}^{-2}\right)$ & Origin \\
\hline Fe II $\lambda 2344$ & $2343.23 \pm 0.15$ & $0.63 \pm 0.11$ & 14.11 & Gal. \\
Fe II $\lambda 2344$ & $2352.87 \pm 0.39$ & $0.59 \pm 0.16$ & 14.08 & M87 \\
Fe II $\lambda 2374$ & $2373.86 \pm 0.28$ & $0.80 \pm 0.24$ & 14.80 & Gal. \\
Fe II $\lambda 2383$ & $2382.22 \pm 0.22$ & $1.13 \pm 0.15$ & 13.94 & Gal. $^{1}$ \\
Fe II $\lambda 2383$ & $2391.09 \pm 0.22$ & $1.33 \pm 0.15$ & 13.04 & M87 \\
Mn II $\lambda 2577$ & $2586.21 \pm 0.52$ & $0.70 \pm 0.48$ & 13.57 & M87 \\
Fe II $\lambda 2587$ & $2586.11 \pm 0.39$ & $0.39 \pm 0.35$ & 14.03 & Gal. \\
Fe II $\lambda 2587$ & $2595.62 \pm 0.36$ & $1.21 \pm 0.21$ & 14.59 & M87 \\
Fe II $\lambda 2600$ & $2599.74 \pm 0.24$ & $0.73 \pm 0.17$ & 13.78 & Gal. \\
Fe II $\lambda 2600$ & $2609.70 \pm 0.26$ & $1.66 \pm 0.16$ & 14.20 & M87 \\
Mn II $\lambda 2606$ & $2606.03 \pm 0.57$ & $0.87 \pm 0.21$ & 13.92 & Gal. \\
Mn II $\lambda 2606$ & $2617.19 \pm 0.78$ & $0.72 \pm 0.19$ & 13.83 & M87 \\
Mg II $\lambda 2796$ & $2795.91 \pm 0.10$ & $1.28 \pm 0.12$ & 13.58 & Gal. ${ }^{3}$ \\
Mg II $\lambda 2796$ & $2803.08 \pm 0.11$ & $1.59 \pm 0.40$ & 13.70 & M87 \\
Mg II $\lambda 2803$ & $2813.61 \pm 0.24$ & $1.91 \pm 0.55$ & 14.05 & M87 \\
Mg I $\lambda 2853$ & $2863.19 \pm 0.35$ & $1.27 \pm 0.18$ & 12.08 & M87 \\
Ca II $\lambda 3935$ & $3949.40 \pm 0.26$ & $2.10 \pm 0.20$ & 13.45 & M87 \\
Ca II $\lambda 3970$ & $3984.29 \pm 0.32$ & $1.83 \pm 0.12$ & 13.80 & M87 \\
Na I $\lambda 5891$ & $5909.44 \pm 2.15$ & $0.61 \pm 0.69$ & 12.48 & M87 \\
Na I $\lambda 5897$ & $5915.01 \pm 1.07$ & $2.96 \pm 0.72$ & 12.53 & M87 \\
\hline
\end{tabular}

${ }^{1}$ Contaminated by M87 Fe II $\lambda 2376$.

2 Contaminated by Galactic Mn II $\lambda 2594$.

${ }^{3}$ Contaminated by Galactic Mn I $\lambda 2795$.

${ }^{4}$ Contaminated by Galactic Mg II $\lambda 2803$ and M87 Mn I $\lambda 2795$. 